\title{
Vorticity analysis within multiple deformable inclusions under pure shear
}

\author{
Ritabrata Dasgupta \\ Department of Geological Sciences, Jadavpur University, Kolkata-700032, India \\ E-mail: ritabrata.dasgupta5@gmail.com
}

\begin{abstract}
This work is aiming at analyzing the vorticity vector in $2 \mathrm{D}$ of deformable inclusions with the help of analytical techniques. The considerations made are first, inclusions are initially spherical, deformable; second, strain distribution within the inclusions are not homogeneous The ratio of inclusion diameter ("a") to mean inter-inclusion distance ("b") that is (a/b) is less than about 0.6 .Considering 'strain rate' as natural strain the rate is infinitesimally small. Vorticity of particles inside the inclusions is also estimated while accounting different competency contrast conditions between matrix and inclusions. It is seen that competency contrast is inversely proportional to the vorticity value. Also after a threshold value the vorticity spin becomes opposite in directional sense. Probable reasons for this hiatus are discussed.
\end{abstract}

Keywords: Deformable Ductile Inclusions, Pure Shear, Vorticity, Viscosity / Competency Contrast.

\section{Introduction}

Previously many workers have shown keen interest in both rigid and deformable as well as single and multiple inclusions under shearing. In the beginning of twenty first century, precisely between (2000 to 2005) some developments on this topic took place. Numerical methods were introduced with previous analogue modelling approaches. It was established the geometry of the inclusion whether rigid or deformable is strongly guided by the heterogeneous flow field in and around inclusions (Samanta et al, 2002). Experimental physical modelling related to this topic and consecutive micro-structural analysis was carried out by structural geologists all over the world (Piazolo and Passchier, 2002) both under simple shear (Samanta , 2003) and in pure shear (Mandal , 2003). There was huge enlightenment on the flow patterns around the inclusions and their emphasis on the deformation (Mandal et al., 2005; Samanta et al., 2003). Also emphasis was shown on both infield and outer-field viscous flow (Jiang, 2006). First ever rotational kinematical approach was made by Mandal. Et al., 2005 but it was about predominantly bulk rotation of the inclusion. Firs approach on far field vorticity was made by Marques et al 2007. As time progressed the numerical approach became more and more advanced (Schmid, 2002; Davis et al, 2013). The vorticity could be determined by marking the particles of the medium in a small neighborhood of the point in question, and watching their relative displacements as they move along the flow. In fluid dynamics, the vorticity is a pseudo-vector field that describes the local spinning motion of a fluid near some point, as would be seen by an observer located at that point and traveling with the fluid. The Vorticity vector would be twice the mean angular velocity vector of those particles about their center of mass, oriented according to the right-hand rule.

\section{Methodology}

Deformation of a particle present within any multiple ductile inclusions under pure shear deformational condition is estimated. The considerations made are: (1) Inclusions are initially spherical, ductile and deformable. (2) The ratio of inclusion diameter ("a") to mean inter-inclusion distance ("b") that is (a/b) is less than about 0.6 . For a given value of " $m$ " (viscosity ratio between inclusion and matrix), strain partitioning between a stiff inclusion and bulk system (i.e. the ratio of their natural extension rates) increases non-linearly with increasing $\mathrm{a} / \mathrm{b}$ ratios and the gradient of increase becomes steeper when the inter-inclusion distance is less than about twice their diameter (i.e. $\mathrm{a} / \mathrm{b}>$ about 0.5 ). The strain distribution within a deformed inclusion is homogeneous when the $\mathrm{a} / \mathrm{b}$ ratio is less than about 0.6 . (3) At the time of expressing 'strain rate' it is considered the shortening took place in negligible time. A system of deformable, spherical inclusions of diameter $2 \mathrm{a}$ within a Newtonian viscous matrix with coherent interfaces is considered (Fig. 1). The inclusions are uniformly distributed with their centres spaced at an interval of $2 b$. The system is subjected to pure shear deformation at a rate $\left(\varepsilon^{*}\right)$. To describe the flow field around an inclusion, a Cartesian coordinate frame is chosen at the centre of an inclusion with the $\mathrm{x}$ axis parallel to the bulk extension direction, $y$ axis along the direction of principal shortening, and $\mathrm{z}$ axis along the direction of no bulk flow (Fig. 1). Lamb's (1932)method of analysis is adopted in this case that expresses the velocity field in terms of solid harmonic functions, and the velocity functions are derived in only two dimensions for flow inside an inclusion in a multiple inclusion system . In the numerical analysis, the velocity functions are considered on a two-dimensional section perpendicular to the direction of no bulk strain. In Fig1 (a) Model of the inclusion-matrix system under theoretical considerations is shown. In Fig1 (b) the consideration of Cartesian and spherical coordinates on the central section of an inclusion normal to the direction of no bulk flow (z axis) is shown; where $\left(\varepsilon^{*}\right)$ : pure 
shear rate of bulk deformation, 2a: diameter of inclusions (shaded) and 2b: average inter-inclusion distance. (After Mandal et al, 2003)

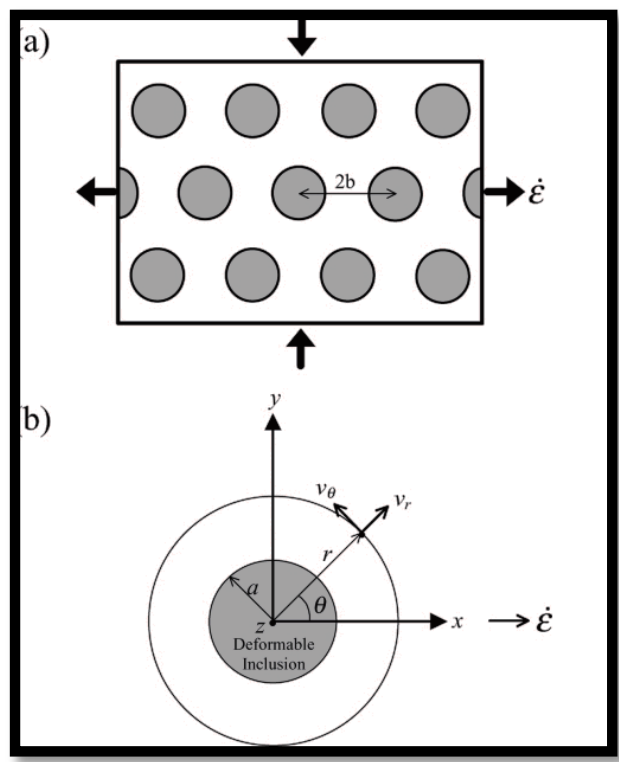

Fig. 1: (A) Model of the Inclusion-Matrix System under Theoretical Considerations. (B) Consideration of Cartesian and Spherical Coordinates on the Central Section of an Inclusion Normal to the Direction of No Bulk Flow (Z Axis). ( $\mathcal{E}^{*}$ ): Pure Shear Rate of Bulk Deformation. 2a: Diameter of Inclusions (Shaded) and 2b: Average Inter-Inclusion Distance. (After Mandal Et Al, 2003)

\section{Equations}

Velocity components of flow inside the inclusions are as follows, In "X" direction- $\mathrm{u}=\frac{\mathrm{A}_{2}}{21} \frac{\mathrm{r}^{2}}{\mathrm{a}^{2}}\left(5-2 \frac{\mathrm{x}^{2}-\mathrm{y}^{2}}{\mathrm{r}^{2}}\right) \mathrm{x}+2 \mathrm{~B}_{2} \mathrm{X}$.

In "Y" direction- $v=\frac{A_{2}}{21} \frac{r^{2}}{a^{2}}\left(-5-2 \frac{x^{2}-y^{2}}{r^{2}}\right) y-2 B_{2} y$

And $A_{2}=2 \varepsilon^{\cdot}\left[\left(1+\frac{5}{2} \frac{b^{7}}{a^{7}}\right) L-\frac{21}{4}\right] \frac{m-1}{L J+I}$

$\mathrm{B}_{2}=\frac{1}{2} \varepsilon^{\cdot}\left[1+\left(\frac{\mathrm{m}-1}{\mathrm{LJ}+\mathrm{I}}\right)\left(\frac{5}{2}-\frac{\mathrm{a}^{3}}{\mathrm{~b}^{3}}-\frac{\mathrm{b}^{7}}{\mathrm{a}^{7}} \mathrm{~L}\right)\right]$

Again, $\mathrm{I}=1+\frac{3}{2} \mathrm{~m}+(\mathrm{m}-1) \frac{\mathrm{a}^{3}}{\mathrm{~b}^{3}}$

$J=\left[\frac{16}{21}\left\{(1-m)-\frac{b^{7}}{a^{7}}(m+1)\right\}-\frac{1}{7} \frac{b^{7}}{a^{7}} m\right]$
$\left.\mathrm{L}=\frac{21\left(4+\frac{19}{4} \mathrm{~m}\right)}{\left[19(\mathrm{~m}-1)+\frac{\mathrm{b}^{7}}{2 \mathrm{a}^{7}}(80+95 \mathrm{~m})\right.}\right]$.

(Mandal et al, 2003)

Where $\mathrm{r}^{2}=\mathrm{x}^{2}+\mathrm{y}^{2}$ and $\mathrm{a}$ is the radius of inclusion. $\mathrm{A}_{2}, \mathrm{~B}_{2}, \mathrm{I}, \mathrm{J}, \mathrm{L}$ are constants, the expressions for which involve the ratio of inclusion diameter and mean inter- inclusion distance $(\mathrm{a} / \mathrm{b})$ and the viscosity ratio between inclusion and matrix (m) [Eq.(iii) to Eq.(vii) ] . The velocity functions in Equation (i) and (ii) reflect the nature of deformation of an inclusion in inclusion-matrix systems. In these equations, $A_{2}$ becomes nearly zero when the $a / b$ ratio is extremely low, indicating a homogeneous deformation inside the inclusion as in the case of a single inclusion system (cf. Eshelby, 1957; Gay, 1968a). But, $A_{2}$ becomes a non-zero quantity for higher values of $a / b$, implying that the deformation is heterogeneous when the inclusions are closer to one another in the system.

This work estimates the vorticity along the radius of the inclusion under pure shear conditions and to show how it varies according to different assigned values of viscosity ratio between inclusion and matrix $(\mathrm{m})$.

The working formula for vorticity calculation is very simple.

$\mathrm{w}=\frac{1}{2}\left(\frac{\mathrm{du}}{\mathrm{dx}}-\frac{\mathrm{dv}}{\mathrm{dy}}\right)$

Where $\mathrm{w}=$ vorticity vector

\section{Results}

In this work vorticity along the radius of the inclusion is estimated under pure shear compression with varying viscosity ratio between matrix and inclusions of the system. The " $m$ " value assigned is 5 , 10, 20 and 40. In Fig 2 the fluctuation of the vorticity vector along the radius of an inclusion with respect to different viscosity ratios " $\mathrm{m}$ " is monitored. When " $\mathrm{m}$ " value is lower (5-10) the vorticity is positive but when " $m$ " value is relatively larger (20-40) then the vorticity becomes negative or opposite to that of previous case. From the graph of Fig 2 the change in vorticity values with respect to different " $m$ " values are clearly seen. The fluctuation of vorticity value along the radius of a deformable inclusion for a fixed " $\mathrm{m}$ " value is very low which is evident from the figure. In the figure variation of vorticity value is shown with respect to incremental change of the polar co-ordinate of the system(r). In this case vorticity within the inclusion is calculated, so " $\mathrm{r}$ " value is taken in such small increment and up to limited extent.

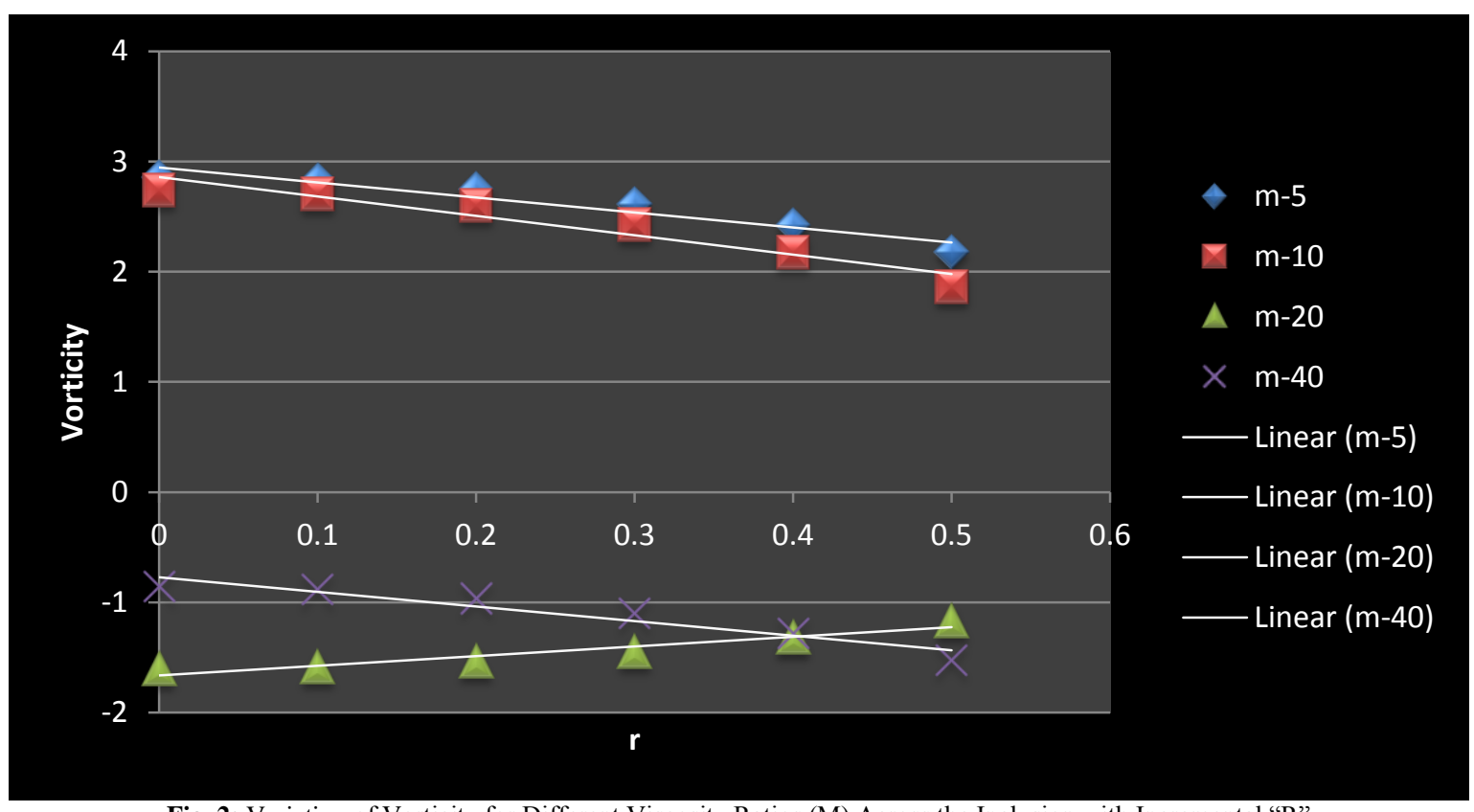

Fig. 2: Variation of Vorticity for Different Viscosity Ratios (M) Across the Inclusion with Incremental "R" 


\section{Conclusion}

From this analytical work several inferences can be drawn from the graphical representations. Firstly, change in vorticity with increasing radius is low as indicated by the gentle slopes of the given curves. Secondly, vorticity decreases with increasing radius of the inclusion although there might be an exception if competency contrast is very high. Thirdly, two opposite senses of rotation are observed. Lastly the overall value of vorticity increases as the viscosity ratio between inclusion and matrix (m) decreases although there might be any departure from this behavior at very high values of ' $\mathrm{m}$ '. The change in rotational sense with increasing viscosity contrast between matrix and inclusion " $\mathrm{m}$ " can be subjected by occurrence of prominent change in bulk rheology of the block with increasing " $\mathrm{m}$ ". At lower values of " $\mathrm{m}$ " the vorticity value is positive which implies that the velocity gradient along " $\mathrm{X}$ " axis is higher than " $\mathrm{Y}$ " axis. Inversely at higher values of " $\mathrm{m}$ " the values of vorticity become negative which implies that velocity gradient along " $Y$ " axis exceeds the value of " $X$ " axis. These two exactly opposite behaviour of material can only be justified by rheological terms as it results due to change of response of the material under equal stress. So change in bulk rheology depending upon the value of " $\mathrm{m}$ " can only be the controlling factor for different response of apparently similar material under same stress. But it is to be mentioned that the limitation of this study is that the (a/b) ratio should be always less than 0.6 which indicates a compact packing of inclusions.

\section{Acknowledgements}

I acknowledge the facilities provided by Department of Geological Sciences, Jadavpur University, Kolkata, India. I also acknowledge Debija Chakraborty (IIT Roorkie), Debattam Sarkar (Jadavpur University, Dept. of Geological Sciences) and Saheli Chowdhury (Jadavpur University, Dept of Physics) for their valuable comments and scientific discussions about this work. Saheli Chowdhury also helped me in mathematical calculations related to this work.

\section{References}

[1] Pizzalo S., Passchier C.W., 2002, Experimental modelling of viscous inclusions in a circular high-strain shear rig: Implications for the interpretation of shape fabrics and deformed enclaves, Journal of Geophysical Research, VOL. 107, NO. B10, 2242, doi: 10.1029/2000JB000030 http://dx.doi.org/10.1029/2000JB000030.

[2] Schmid D.W., 2002, Finite and infinite heterogeneities under pure and simple shear, Doctoral Thesis submitted to SWISS FEDERAL INSTITUTE OF TECHNOLOGY ZURICH

[3] Davis J.R., Titus J.S., Horsman E.,2013, Non-steady homogeneous deformations: Computational techniques using Lie theory, and application to ellipsoidal markers in naturally deformed rocks, Journal of Structural Geology, Vol-56 (2013), 142-155 http://dx.doi.org/10.1016/j.jsg.2013.08.003.

[4] Dazhi Jiang, 2006, Numerical modelling of the motion of deformable ellipsoidal objects in slow viscous flows, Journal of Structural Geology, doi:10.1016/j.jsg.2006.09.009 http://dx.doi.org/10.1016/j.jsg.2006.09.009.

[5] Mandal N., Samanta S.K., Bhattacharyya G., Chakraborty C.,2005, Rotation behaviour of rigid inclusions in multiple association: insights from experimental and theoretical models, Journal of Structural Geology,27 (2005),679-692 http://dx.doi.org/10.1016/j.jsg.2004.11.007.

[6] Mandal N., Samanta S.K., Bhattacharyya G.,Chakraborty C.,2003, Deformation of ductile inclusions in a multiple inclusion system in pure shear, Journal of Structural Geology,25 (2003),1359-1370 http://dx.doi.org/10.1016/S0191-8141(02)00204-3.

[7] Mandal N., Samanta S.K., Chakraborty C., 2005, Numerical models of flow patterns around a rigid inclusion in a viscous matrix undergoing simple shear: implications of model parameters and boundary conditions, Journal of Structural Geology, 27 (2005), 1599-1609 http://dx.doi.org/10.1016/j.jsg.2005.05.003.
[8] Marques F.O., Schmid D.W., Anderson T.B., 2007, Applications of inclusion behaviour models to a major shear zone system: The Nordfjord-Sogn Detachment Zone in western Norway, Journal of Structural Geology, $29 \quad$ (2007), $\quad 1622-1631$ http://dx.doi.org/10.1016/j.jsg.2007.05.008.

[9] Samanta S.K.,Mandal N., Chakraborty C.,2002, Development of structures under the influence of heterogeneous flow field around rigid inclusions: insights from theoretical and numerical models, EarthScience Reviews, 58 (2002),85-119 http://dx.doi.org/10.1016/S00128252(01)00084-8.

[10]Samanta S.K., Mandal N., Chakraborty C., 2003, Flow patterns around rigid inclusions in a multiple inclusion system undergoing bulk simple shear deformation, Journal of Structural Geology, 25 (2003),209-221 http://dx.doi.org/10.1016/S0191-8141(02)00032-9.

[11]Gay, N.C., 1968a, Pure shear and simple shear deformation of inhomogeneous viscous fluids-I. Theory., Tectonophysics 5, 211-234. http://dx.doi.org/10.1016/0040-1951 (68)90065-6.

[12]Eshelby, J.D., 1957, the determination of the elastic field of an ellipsoidal inclusion, and related problems, Proceedings of the Royal Society of London A241, 376-396. http://dx.doi.org/10.1098/rspa.1957.0133.

[13]Lamb, H., 1932, Hydrodynamics, Cambridge University Press, Cambridge.

[14]Dasgupta, R., 2014, Kinematics of a multiple deformable inclusion system under pure shear, 4th International Science Congress, Udaipur.

[15] Happel, J., 1957. Viscosity of suspensions of uniform spheres. Journal

of Applied Physics 28, 1288-1292. http://dx.doi.org/10.1063/1.1722635. 